\title{
Olas de calor e influencia urbana en Madrid y su área metropolitana
}

\section{Heat waves in urban and suburban Madrid}

\author{
Fernández García, F.* \\ Rasilla Álvarez, D.*
}

\section{INTRODUCCIÓN}

Las previsiones del último informe del IPCC indican que el calentamiento observado desde el pasado siglo, se mantendrá a lo largo de éste, al tiempo que aumentarán los días muy cálidos y las olas de calor. En las ciudades el aumento térmico se verá incrementado como consecuencia de la isla de calor y sus efectos serán especialmente graves sobre la salud y el bienestar de los habitantes. Diversos estudios muestran que las olas de calor provocan un incremento de las tasas de ingresos hospitalarios y un aumento de la mortalidad, y, según algunos autores (Coppe et al. y Taha et al., 2004) estos periodos cálidos, junto con la contaminación atmosférica, son los mayores riesgos de origen natural en las áreas urbanas.

En las últimos años se observa, además, un aumento notable del consumo eléctrico, derivado del uso de los aparatos de aire acondicionado y los picos de consumo son cada vez más frecuentes durante el verano, en clara coincidencia con el aumento de la temperatura (Valor et al., 2001). Todo ello determina que la contaminación procedente del consumo doméstico

* Grupo de investigación GEOCLIMA. Universidad Autónoma de Madrid (felipe.fernandez@uam.es). Universidad de Cantabria (domingo.rasilla@unican.es).

Este trabajo se ha desarrollado en el marco del proyecto S2007/HUM-0474, financiado por la Comunidad de Madrid en el Programas de actividades de I+D entre Grupos de Investigación, en su edición de Socioeconomía, Humanidades y Derecho. 
haya aumentado hasta ocupar el segundo lugar, tras el tráfico rodado, en el total de emisiones urbanas. En el caso concreto de España, representan entre el $5 \%$ y el $10 \%$ del total de emisiones de $\mathrm{CO}_{2}$ y su tendencia crece de forma continua (MMA, 2007).

Tras la ola de calor de 2003 se han generalizado las medidas tendentes a paliar los efectos de las olas de calor por parte de las autoridades nacionales, regionales y locales (Informe especial del IPCC, 2007, García, J. C. y Alberdi, J. C., 2004; Fischer et al., 2004). Así, numerosas ciudades han puesto en funcionamiento sistemas de alerta, siguiendo el modelo desarroIlado por Kalstein y colaboradores (Ebi et al., 2004; Sheridan et al., 2004; Kalkstein et al., 2008), cuya aplicación, primero en Filadelfia en 1995 y después en otras ciudades, ha demostrado los beneficios en la prevención del exceso de mortalidad por temperaturas extremas. La Unión Europea ha puesto en marcha programas como el EURO HEAT, cuyo objetivo es la mejora de las medidas de sanidad pública en caso de condiciones meteorológicas extremas y olas de calor; PHEWE, para la prevención de efectos agudos para la salud de las condiciones meteorológicas en Europa y el CASHh con objeto de estudiar el cambio climático y estrategias de adaptación para la salud humana en Europa. La Agencia Espacial Europea (ESA), por su parte, ha realizado una campaña de medidas en Madrid durante los meses de junio y julio de 2008, como avance del proyecto "Urban heat island and urban thermography", que se desarrollará en 10 ciudades europeas durante los próximos años (DESIREX, 2008; http://www.esa.int/esaLP/).

\section{Calentamiento global y olas DE CAlor EN EsPaÑa}

En España el aumento de las temperaturas es similar, o incluso mayor, al observado en el resto del continente europeo y las proyecciones futuras estiman que el calentamiento afectará a todas las áreas, pero será más acusado en el centro y este peninsular (MMA, 2007 y 2008). Las medidas para afrontar los impactos de estas tendencias se recogen en el "Plan Nacional de Adaptación frente al cambio climático" donde se señala que las estrategias de ordenación del territorio y los planes urbanísticos deben prestar una atención especial a la información climática, para la elaboración de la normativa técnica sobre edificación. Resultado de esta política ha sido la aprobación del Código Técnico de la Edificación, en el que se incluye el clima regional como factor fundamental para el desarrollo de una arquitectura energéticamente sostenible, basada en el ahorro y la eficiencia energética (RD 314-2006). 
Por su parte, los gobiernos autonómicos en colaboración con la Agencia Estatal de Meteorología (AEMET) han desarrollado sistemas de predicción y alerta a la población frente a las olas de calor. En el caso concreto de la Comunidad de Madrid, los estudios previos se iniciaron en 2004 y está en funcionamiento desde 2006.

En este contexto presentamos nuestro trabajo, cuyo objetivo principal se centra en evaluar las influencia de la ciudad de Madrid durante lo episodios de ola de calor y extremos térmicos. Sigue la línea de investigación sobre el clima urbano de Madrid, iniciada en 1984 (López Gómez y Fernández García, 1984) y al que han seguido otros sobre la isla de calor, el confort y la contaminación atmosférica en Madrid y su área metropolitana (López Gómez et al., 1993, Fernández García et al., 2003, Fernández García, 2001-2002 y 2005). Asimismo, constituye un avance de otros estudios ya iniciados en los que se analizan otros aspectos relacionados con el tema.

\section{El área de estudio: interés y características}

El área metropolitana madrileña es una zona muy transformada por la acción antrópica, con una gran concentración de población en torno a Madrid, la mayor ciudad de España, y con un clima mediterráneo continental muy extremado en el que las olas de calor son muy frecuentes

Situada en la cuenca sedimentaria del Tajo, el relieve presenta formas suaves labradas por los ríos Jarama, Henares y Manzanares en los materiales blandos de la cuenca y el paisaje ha sufrido importantes transformaciones como consecuencia de la fuerte presión demográfica. La vegetación natural ha sido sustituida por cultivos o está muy modificada y las masas que hoy se conservan sólo son los retazos de comunidades antaño más extensas. Las principales masas vegetales se localizan al norte y noroeste, destacando el Monte del Pardo y la Casa de Campo, gran parque suburbano próximo a la ciudad; todas ellas representativas del ecosistema mediterráneo, colonizadas por encinares muy transformados y sustituidos en muchos puntos por comunidades arbustivas.

La fuerte concentración de población es el rasgo más destacado de esta zona, donde se localiza Madrid con más de tres millones de habitantes y un conjunto de núcleos próximos con poblaciones que oscilan entre los 70.000 y más de 150.000 habitantes. En total más de 5.000 .000 de personas viven en un radio inferior a $50 \mathrm{~km}$ en torno a la capital, de los cuales los grupos de riesgo integrados por los menores de 5 años y los mayores de 65, superan el millón de personas, en torno al 20\% de la población total (cuadro 1). 
Cuadro 1. Población en Madrid y Área metropolitana

Población total Menores de $5 \quad \% \quad$ Más de $65 \quad \%$

\begin{tabular}{|c|c|c|c|c|c|}
\hline Comunidad de Madrid & 6.081 .689 & 340.674 & 5,6 & 866.031 & 14,2 \\
\hline Madrid & 3.132 .463 & 151.077 & 4,8 & 587.682 & 18,8 \\
\hline AME & 538.447 & 32.378 & 6,0 & 45.349 & 8,4 \\
\hline AMN & 255.969 & 15.587 & 6,1 & 22.860 & 8,9 \\
\hline $\mathrm{AMO}$ & 208.061 & 17.529 & 8,4 & 15.556 & 7,5 \\
\hline AMS & 1.046 .465 & 59.653 & 5,7 & 102.778 & 9,8 \\
\hline Total Madrid y AM & 5.181 .405 & 276.224 & 5,3 & 774.225 & 14,9 \\
\hline
\end{tabular}

Fuente: Elaboración propia a partir de los datos del Instituto de Estadística de la Comunidad de Madrid, año 2007.

El clima presenta los rasgos típicos de una zona mediterránea continental, como son las escasas precipitaciones, una elevada insolación y fuertes amplitudes térmicas, con inviernos fríos y veranos muy cálidos. Las situaciones anticiclónicas representan más del $57 \%$ del total de los días del año y más del $70 \%$ durante el verano, periodo en el que el anticiclón de las Azores mantiene una gran estabilidad atmosférica, con vientos flojos o en calma y una fuerte insolación. Esto explica que las temperaturas medias de las máximas se sitúen por encima de los $28^{\circ} \mathrm{C}$ durante los tres meses de verano y que exista un número de días relativamente alto en los que las temperaturas superan los $34^{\circ} \mathrm{C}$, valor del percentil 90. Las mínimas medias oscilan entre los $12,7^{\circ} \mathrm{C}$ de septiembre y los $16,1^{\circ} \mathrm{C}$ de julio; en este mes y en el siguiente el $5 \%$ de los días pueden superar los $20^{\circ} \mathrm{C}$ (cuadro 2 ).

Cuadro 2. Temperaturas estivales en el aeropuerto de Barajas (1961-2004)

\begin{tabular}{lcccc} 
& \multicolumn{3}{c}{ Temperaturas máximas } \\
& Junio & Julio & Agosto & Septiembre \\
\hline Media & 28,5 & 32,8 & 32,3 & 27,5 \\
P90 & 34,4 & 37,2 & 36,5 & 33,2 \\
P95 & 35,7 & 38,0 & 37,5 & 34,4 \\
P98 & 37,0 & 39,2 & 38,6 & 35,4 \\
& & & & \\
\hline & & & \\
& & & \\
& & & & \\
\hline Media & Julio & Agosto & Septiembre \\
P90 & 13,2 & 16,1 & 15,9 & 12,7 \\
P95 & 17,2 & 19,4 & 19,3 & 16,6 \\
P98 & 18,0 & 20,0 & 20,2 & 17,4 \\
& 19,1 & 21,0 & 21,0 & 18,2 \\
\hline
\end{tabular}




\section{Metodología Y FUENTES}

El trabajo se estructura en tres apartados: en el primero analizamos la frecuencia de las olas de calor y de los días cálidos en el área metropolitana madrileña, como indicativos del clima regional; en el segundo estudiamos la isla de calor en verano y la influencia de la ciudad en la intensificación y duración de los episodios cálidos y en el tercero se evalúa la influencia de los diferentes usos y estructuras urbanas existentes dentro de la ciudad.

Los datos climáticos constituyen la base fundamental del trabajo, pero se han utilizado además el mapa de usos de suelo del proyecto CORINE, de la Comunidad de Madrid y mapas vectoriales de barrios y distritos de Madrid. La herramienta de integración ha sido el SIG comercial ArcGis 9.2.

Las series climáticas analizadas proceden de los datos registrados en el observatorio de Barajas y en los observatorios de la red municipal, instalada por el Ayuntamiento de Madrid en 2002. El primero está situado en el aeropuerto de Madrid y lo hemos considerado representativo de las características del clima regional; la red municipal está formada por siete estaciones localizadas dentro de la ciudad, pero ocupando espacios de morfología urbana diferenciada, tal y como se muestra en el mapa 1.

MAPA 1. LOCALIZACIÓN DE OBSERVATORIOS METEOROLÓGICOS Y PRINCIPALES USOS DEL SUELO EN EL ÁREA METROPOLITANA DE MADRID

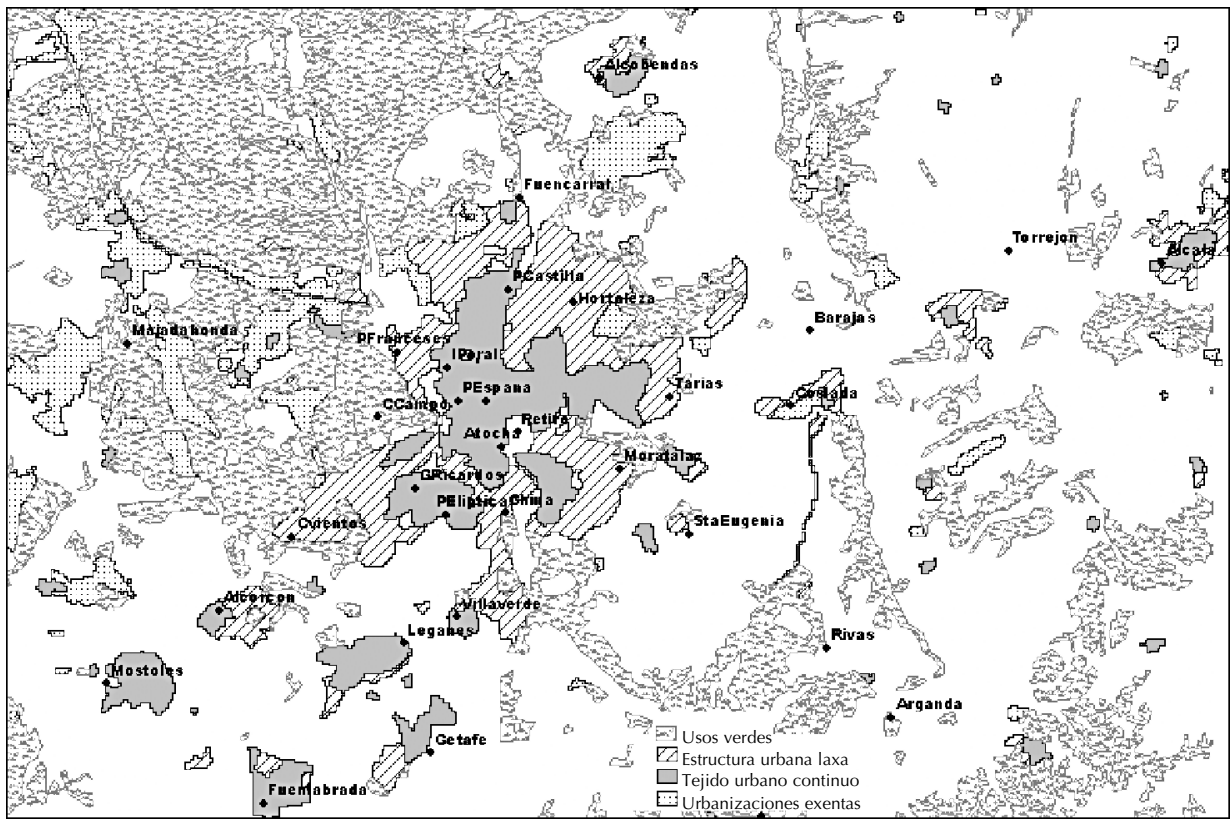


Adicionalmente, hemos utilizado los datos de otros tres observatorios completos (Torrejón, Getafe y Cuatro Vientos) situados en aeropuertos o aeródromos de la periferia urbana y pertenecientes a la red meteorológica nacional dependiente de la EMET (Agencia española de meteorología, anteriormente INM) y 16 observatorios de la red de vigilancia y control de la contaminación del Ayuntamiento de Madrid y de la Comunidad. Las características de cada uno de ellos aparecen en el cuadro 3.

\section{CuAdro 3. ObSERVATORIOS METEOROLÓGICOS: LOCALIZACIÓN Y CARACTERÍSTICAS} GENERALES

\begin{tabular}{|c|c|c|c|c|c|c|}
\hline Estación & Ind & ALT & $x$ & Y & Red & Periodo \\
\hline Barajas aeropuerto & 329 & 582 & 453857.1 & 4478386.8 & AEMET & 1961-2008* \\
\hline Fuencarral & 100 & 729 & 442167.7 & 4483740.9 & Municipal & 2002-2004 \\
\hline Barceló & 105 & 636 & 440791.9 & 4475573.5 & Municipal & 2002-2004 \\
\hline Hortaleza & 107 & 704 & 444325.3 & 4479500.0 & Municipal & 2002-2004 \\
\hline La China & 104 & 566 & 441570.5 & 4471049.2 & Municipal & 2002-2004 \\
\hline Moratalaz & 102 & 687 & 446193.6 & 4472819.2 & Municipal & 2002-2004 \\
\hline Puente de los Franceses & 106 & 587 & 437242.9 & 4477520.8 & Municipal & 2002-2004 \\
\hline Torre Arias & 101 & 640 & 448185.5 & 4475741.2 & Municipal & 2002-2004 \\
\hline Villaverde & 103 & 594 & 439630.9 & 4466862.8 & Municipal & 2002-2004 \\
\hline Estación & & ALT & $x$ & Y & Red & Periodo \\
\hline Cuatro Vientos aeródromo & & 687 & 433006.0 & 4470064.0 & AEMET & 2003 \\
\hline Getafe base aérea & & 617 & 438595.3 & 4461382.4 & AEMET & 2003 \\
\hline Retiro & & 667 & 442489.6 & 4473750.9 & AEMET & 2003 \\
\hline Torrejón base aérea & & 611 & 461835.0 & 4481579.5 & AEMET & 2003 \\
\hline Atocha & & 629 & 441430.3 & 4473724.9 & Cont. Madrid & 2003 \\
\hline Casa de Campo & & 645 & 436466.8 & 4474906.3 & Cont. Madrid & 2003 \\
\hline Cuatro Caminos & & 699 & 440209.9 & 4477342.4 & Cont. Madrid & 2003 \\
\hline General Ricardos & & 581 & 437985.0 & 4472037.0 & Cont. Madrid & 2003 \\
\hline Isaac Peral & & 672 & 439240.3 & 4476887.6 & Cont. Madrid & 2003 \\
\hline Plaza de Castilla & & 729 & 441715.0 & 4480013.3 & Cont. Madrid & 2003 \\
\hline Plaza de España & & 637 & 439677.3 & 4475558.2 & Cont. Madrid & 2003 \\
\hline Plaza Fdez. Ladreda & & 605 & 439201.2 & 4470971.3 & Cont. Madrid & 2003 \\
\hline Santa Eugenia & & 652 & 448972.4 & 4470185.4 & Cont. Madrid & 2003 \\
\hline Alcalá de Henares & & 595 & 467978.0 & 4481088.0 & Cont. CAM & 2003 \\
\hline Alcobendas & & 688 & 445404.0 & 4488527.0 & Cont. CAM & 2003 \\
\hline Alcorcón & & 709 & 430102.0 & 4467100.0 & Cont. CAM & 2003 \\
\hline Coslada & & 602 & 453061.0 & 4475370.0 & Cont. CAM & 2003 \\
\hline Fuenlabrada & & 699 & 431848.0 & 4459344.0 & Cont. CAM & 2003 \\
\hline Leganés & & 676 & 437499.0 & 4465801.0 & Cont. CAM & 2003 \\
\hline Majadahonda & & 630 & 426409.0 & 4477866.0 & Cont. CAM & 2003 \\
\hline Móstoles & & 660 & 425543.0 & 4464183.0 & Cont. CAM & 2003 \\
\hline
\end{tabular}


Las variables analizadas han sido los máximos y mínimos diarios de temperatura, desde 1961 hasta el 15 de agosto de 2008, en la serie de Barajas; los datos horarios de temperatura durante el periodo 2002-2004, de la red municipal y los datos diarios de estas mismas variables de las redes de contaminación del Ayuntamiento y de la Comunidad, durante la ola de calor de agosto de 2003. Previo a su utilización, las series han sido sometidas a un proceso de control de calidad, eliminando los datos anómalos y completando las lagunas existentes, mediante la correlación entre las series originales y la de Barajas.

\section{Olas de calor y días cálidos en el área metropolitana madrileña}

Caracterización. El concepto de ola de calor lleva implícito el riesgo provocado por temperaturas anormalmente elevadas, por ello su caracterización se basa en dos factores: uno puramente climático, como es la superación de un determinado umbral térmico, que le confiere al registro concreto el carácter de extremo; el segundo hace referencia a los impactos negativos que tales extremos provocan sobre la población. En Madrid, diversos estudios epidemiológicos (García Herrera et al., 2005; Díaz et al., 2006) muestran que la mortalidad aumenta hasta en un $20 \%$ cuando las temperaturas máximas diarias superan los $38{ }^{\circ} \mathrm{C}$, o con una acumulación de al menos cinco días de temperaturas superiores a $36,5{ }^{\circ} \mathrm{C}$. En función de ello, el Sistema de Alerta frente a las olas de calor establece cuatro niveles de riesgo (CAM, 2006):

- Nivel 0, situación de normalidad. Se considera que no existe un incremento de riesgo cuando la temperatura máxima prevista para los próximos cinco días es inferior a los $36,5^{\circ} \mathrm{C}$. Esta situación expresa la "normalidad" de la temperatura estival en la Comunidad de Madrid.

- Nivel I, situación de precaución. Se considera que existe un nivel moderado de riesgo con un perfil bajo de intervención cuando la temperatura máxima prevista para los próximos cinco días es igual o superior a $36,6^{\circ} \mathrm{C}$ e inferior a 38,6 con una duración que no supere los tres días consecutivos.

- Nivel II, alto riesgo. Cuando la temperatura máxima prevista para los próximos cinco días es igual o superior a $38,6^{\circ} \mathrm{C}$ al menos en un día, o cuando se producen al menos cuatro días consecutivos con temperaturas superiores o iguales a $36,6{ }^{\circ} \mathrm{C}$. 
En nuestro estudio hemos considerado dos indicadores del calor extremo estival en Madrid: días cálidos, aquellos en los que la máxima en Barajas ha sido superior a $36,5^{\circ} \mathrm{C}$ y olas de calor cuando esta temperatura se ha mantenido durante más de cuatro días consecutivos, o durante cuatro días, siempre y cuando en alguno de ellos se superen los $38^{\circ} \mathrm{C}$.

Frecuencia y evolución de los días extremadamente cálidos en Barajas

Las características del clima regional, que hemos expuesto anteriormente, determinan que la probabilidad de que aparezcan días extremadamente cálidos es muy alta, de tal modo que en el periodo 1961-2007, en todos los años se ha registrado algún día con temperaturas máximas superiores a $36,5^{\circ} \mathrm{C}$, situándose el máximo de 25 días en lo años 1991 y 2006 (gráfico 1). Por encima de $38{ }^{\circ} \mathrm{C}$, la frecuencia es ligeramente inferior y los máximos han sido de 15 días el año 1991 y 11 en 2005 (gráfico 2).

Gráfico 1. Total de días CON temperaturas máximas en Barajas SUPERIORES A $36,5{ }^{\circ} \mathrm{C}$, DURANTE LOS MESES DE JUNIO, JULIO, AGOSTO Y SEPTIEMBRE (1961-2007)

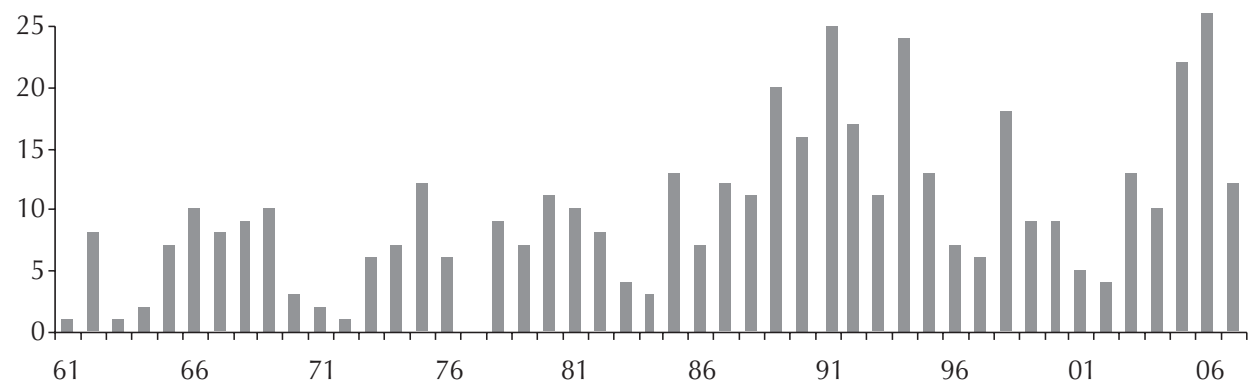

Fuente: Elaboración propia a partir de datos del observatorio de Barajas suministrados por la AEMET.

Gráfico 2. Total de días con temperaturas máximas en Barajas SuPERIORES a $38{ }^{\circ} \mathrm{C}$, DURANTE LOS MESES DE JUNIO, JULIO, AGOSTO Y SEPTIEMBRE (1961-2007)

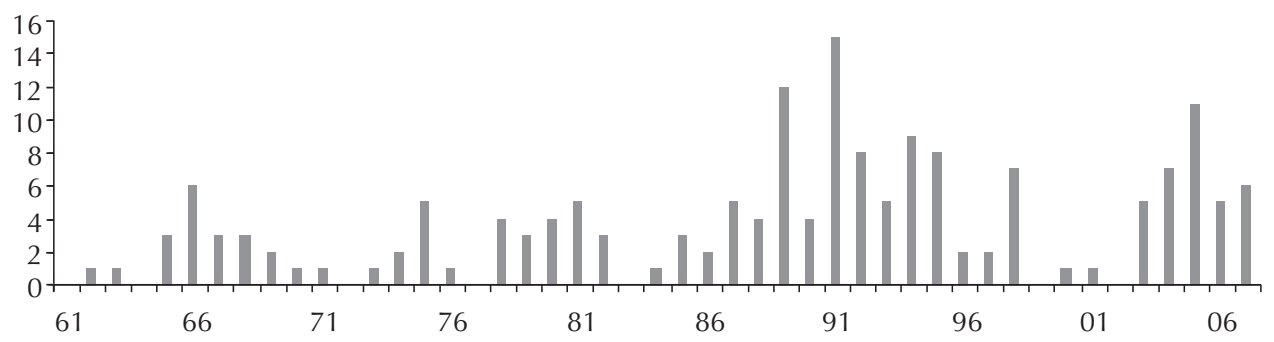

Fuente: Elaboración propia a partir de datos del observatorio de Barajas suministrados por la AEMET. 
La mayor parte de estos eventos extremos se producen en julio y agosto, meses en los que más del $50 \%$ de los años han alcanzado temperaturas máximas superiores a los $38^{\circ} \mathrm{C}$ y más del $80 \%$ han superado los $36,5{ }^{\circ} \mathrm{C}$ (gráfico 3). En junio la probabilidad es bastante menor y en septiembre, es muy raro que se registren máximas superiores a los $36,5^{\circ} \mathrm{C}$.

Gráfico 3. Porcentaje de años en los que se han superado los Valores extremos EN BARAJAS, DURANTE LOS MESES ESTIVAlES (1961-2007)

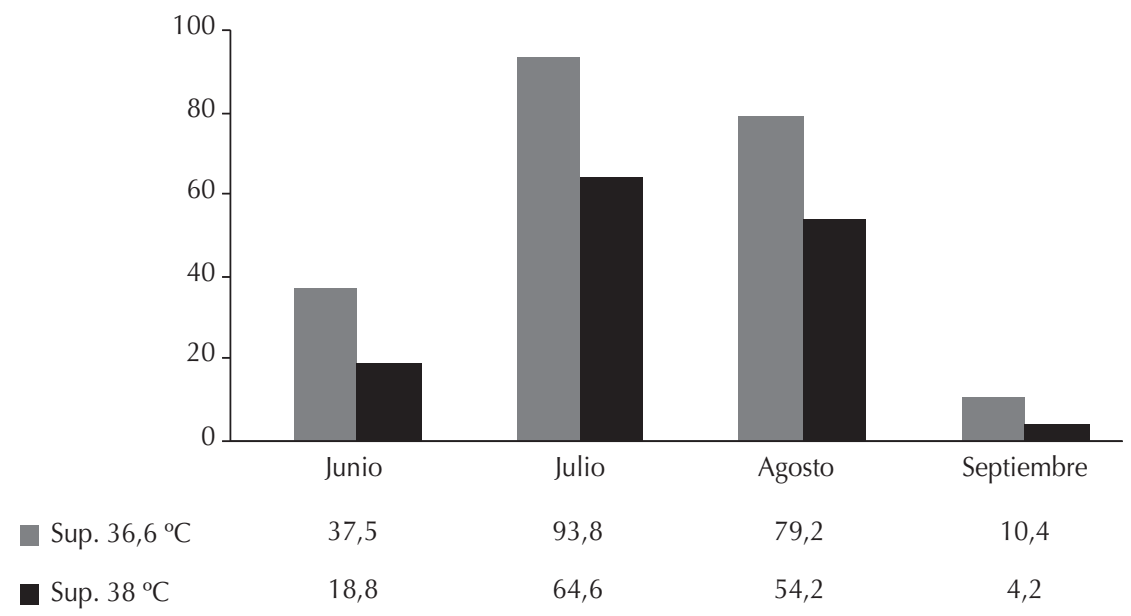

Fuente: Fernández-García, F., a partir de datos de Barajas.

Frecuencia y evolución de las olas de calor en Barajas

La alta frecuencia de estos días extremadamente cálidos constituye, por tanto, un rasgo típico del clima de nuestra zona, pero no indican olas de calor. Para que éstas se produzcan es preciso que se mantengan durante cuatro o más días consecutivos, lo que sucede habitualmente cuando una dorsal en la corriente de altura, combinada con una baja térmica en el centro y oeste peninsular permite la penetración de masas de aire muy cálidas, procedentes del norte de África (Rasilla Álvarez, D. y Fernández García, F., 2005; García Herrera et al., 2002 y 2005).

En el intervalo temporal que va desde el año 1961 hasta el 15 de agosto de 2008 se han registrado cuarenta y siete olas de calor de diferente duración e intensidad (cuadro 4). La media de las máximas en cada uno de esos episodios superaron los $37^{\circ} \mathrm{C}$ y los valores absolutos sobrepasaron 
frecuentemente los $40^{\circ} \mathrm{C}$. La duración media ha sido de 5,7 días, aunque se han producido episodios de diez u once días en los años 1991, 1997, 2004 y 2007; el episodio más largo ha sido de 14 días y se registró en agosto de 2003.

Cuadro 4. Relación de ola de Calor, Registradas en Barajas, entre 1961 y 2008

\begin{tabular}{|c|c|c|c|c|}
\hline Año & Mes & Duración & T. media & T. máxima \\
\hline 1962 & Agosto & 6 & 37,4 & 38 \\
\hline 1966 & Agosto & 5 & 37,9 & 38,6 \\
\hline 1967 & Julio & 6 & 37,8 & 39,8 \\
\hline 1968 & Junio y Julio & 5 & 37,3 & 38,7 \\
\hline 1969 & Julio & 4 & 37,1 & 38 \\
\hline 1969 & Julio & 7 & 37,6 & 39 \\
\hline 1973 & Agosto & 4 & 37,5 & 38,2 \\
\hline 1975 & Julio & 4 & 38 & 38,4 \\
\hline 1976 & Agosto & 4 & 38 & 39,8 \\
\hline 1978 & Julio & 4 & 39,2 & 40,2 \\
\hline 1981 & Junio & 4 & 38 & 39,2 \\
\hline 1982 & Julio & 4 & 38,5 & 39,2 \\
\hline 1985 & Julio & 5 & 37,5 & 38,8 \\
\hline 1988 & Septiembre & 4 & 39 & 40,2 \\
\hline 1989 & Julio & 9 & 38,6 & 41,2 \\
\hline 1989 & Julio y agosto & 8 & 38,3 & 41 \\
\hline 1990 & Julio & 5 & 37,2 & 37,6 \\
\hline 1990 & Julio & 6 & 37,7 & 39,9 \\
\hline 1991 & Julio & 10 & 38,4 & 40 \\
\hline 1991 & Agosto & 4 & 39,6 & 41,2 \\
\hline 1991 & Agosto & 9 & 38,7 & 41 \\
\hline 1992 & Julio & 7 & 37,7 & 39,8 \\
\hline 1992 & Agosto & 4 & 38 & 38,2 \\
\hline 1993 & Julio & 8 & 37,4 & 39 \\
\hline 1994 & Junio y julio & 7 & 37,7 & 39,8 \\
\hline 1994 & Julio & 7 & 37,3 & 38,3 \\
\hline 1994 & Julio & 10 & 36,3 & 38 \\
\hline 1994 & Agosto & 6 & 37 & 38 \\
\hline 1995 & Julio & 8 & 39,8 & 41,5 \\
\hline 1996 & Julio & 6 & 37,7 & 38,6 \\
\hline 1998 & Julio & 8 & 37 & 38,6 \\
\hline 1998 & Agosto & 4 & 37,9 & 39,2 \\
\hline 2001 & Junio & 4 & 37,1 & 38 \\
\hline 2003 & Agosto & 14 & 37,3 & 39,8 \\
\hline 2004 & Junio y julio & 5 & 38,5 & 39,4 \\
\hline 2004 & Julio & 4 & 38,1 & 39,6 \\
\hline 2005 & Junio & 6 & 37 & 37,5 \\
\hline 2005 & Julio & 5 & 38 & 38,5 \\
\hline 2005 & Julio & 4 & 38,4 & 39,4 \\
\hline 2005 & Agosto & 4 & 38,9 & 40,7 \\
\hline 2006 & Julio & 10 & 37,5 & 40 \\
\hline
\end{tabular}


Cuadro 4. Relación de ola de Calor, registradas en Barajas, entre 1961 y 2008 (CONT.)

\begin{tabular}{llccc} 
Año & \multicolumn{1}{c}{ Mes } & Duración & T. media & T. máxima \\
\hline 2006 & Julio & 6 & 37,3 & 37,6 \\
2006 & Julio y agosto & 4 & 37,2 & 37,9 \\
2006 & Septiembre & 5 & 37,7 & 38,6 \\
2007 & Julio y agosto & 11 & 37,7 & 39,9 \\
2008 & Julio & 6 & 37,6 & 39,3 \\
2008 & Agosto & 4 & 38,6 & 39,3 \\
\hline
\end{tabular}

$\overline{\text { Fuente: Elaboración propia a partir de datos del observatorio de Barajas suministrados }}$ por la AEMET.

A partir de la década de los noventa, aumenta la frecuencia y duración de las olas de calor, pero su intensidad se mantiene con pequeñas variaciones. Así, entre 1961 y 1970 se registraron seis olas de calor con una duración media de 5,5 días; la década siguiente disminuye el número y la duración, pero la intensidad es mayor, con medias de las máximas superiores a $38{ }^{\circ} \mathrm{C}$ y máximos absolutos superiores a $39{ }^{\circ} \mathrm{C}$ (cuadro 5). A partir de 1981, aumentan el número de olas de calor y su duración, con cinco episodios de más de diez días; tres de ellas entre 2001 y 2008, periodo en el que se sitúa la más larga registrada en los 48 veranos estudiados y que corresponde a la conocida ola de calor de agosto de 2003. La probabilidad de aparición de olas de calor, por tanto, se ha duplicado en los 20 últimos años en clara concordancia con las previsiones derivadas de los diferentes modelos climáticos (Sánchez et al., 2004; MMA, 2005; Kjellström et al., 2007).

Cuadro 5. Características de las olas de CalOr por déCADAS, DeSDe 1961 HASTA EL 15 DE AGOSTO DE 2008

\begin{tabular}{lcccc} 
Década & Total olas & Duración media & T. media & T. máxima \\
\hline $61-70$ & 6,0 & 5,5 & 37,5 & 38,7 \\
$71-80$ & 4,0 & 4,0 & 38,2 & 39,2 \\
$81-90$ & 8,0 & 5,6 & 38,1 & 39,6 \\
$91-2000$ & 13,0 & 7,0 & 37,9 & 39,4 \\
$2001-2008$ & 15,0 & 6,1 & 37,8 & 39,0 \\
$1961-2008$ & 47,0 & 5,7 & 37,9 & 39,2
\end{tabular}

$\overline{\text { Fuente: Elaboración propia a partir de datos del observatorio de Barajas suministrados }}$ por la AEMET. 


\section{Olas de calor e influencia urbana}

Evaluar de qué modo la ciudad modifica el clima regional en estos episodios cálidos es el objetivo esencial de nuestro trabajo y, como señalamos anteriormente, el efecto urbano se manifiesta mediante el denominado fenómeno de isla de calor. Conocer el ritmo y las características de la isla de calor en verano es el paso previo para plantear las cuestiones relativas a la cuantificación del sobrecalentamiento que en la ciudad, sin duda, se produce.

\section{La isla de calor estival}

Madrid es más cálida que las áreas suburbanas próximas debido al efecto de isla de calor, pero éste es un fenómeno que se produce fundamentalmente durante la noche cuando el calor almacenado por el asfalto y los edificios es reemitido a la atmósfera en forma de radiación de onda larga; durante el día la mayor capacidad calorífica de los materiales urbanos y las múltiples sombras provocadas por los edificios mantienen la ciudad más fría que los alrededores, hablándose incluso de isla de frescor (López Gómez et al., 1993). Todo ello explica que la isla de calor diurna, la obtenida a partir de las temperaturas máximas diarias, sea bastante menos intensa y frecuente que en la nocturna, obtenida a partir de las temperaturas mínimas, tal y como aparece en el gráfico 4: durante la noche, prácticamente todos los días, la ciudad es más cálida, mientras que durante el día su número se reduce considerablemente, entre el 20 y $29 \%$ de junio a agosto y el $13 \%$ en septiembre.

Gráfico 4. Frecuencia en \% de la isLa de CAlOR en MADrid, DURANTE los meSES DE VERANO (2002-2004)

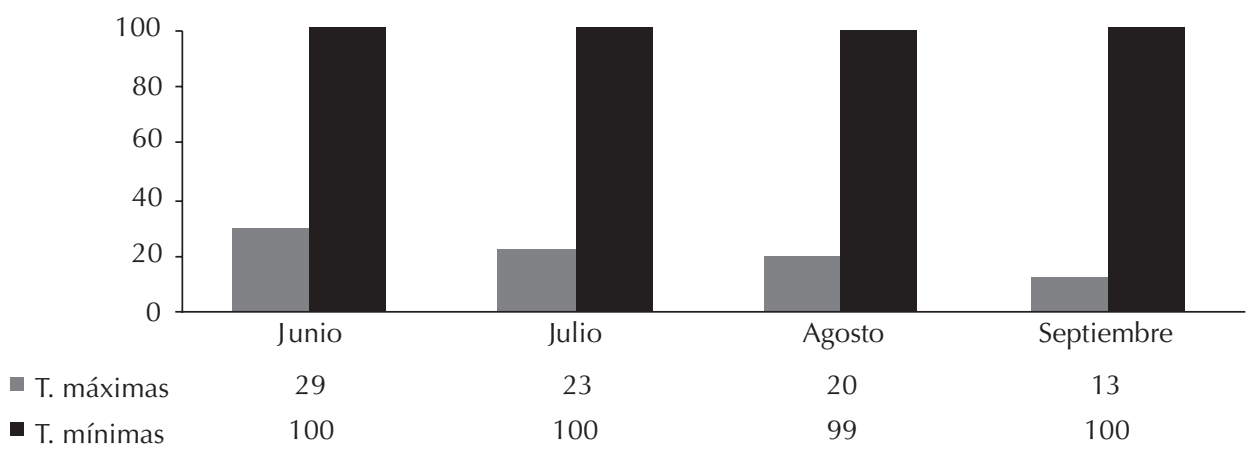

Fuente: Elaboración propia a partir de datos de la red municipal y los del observatorio de Barajas (Tmáximas: Turbmx-TBar; Tmínimas: Turbmin-TBar). 
También varía la intensidad y en las mínimas (gráfico 5A) predominan claramente las fuertes o muy fuertes, con más del $50 \%$ de los casos en los que las diferencias respecto a las zonas no urbanas superan los $6{ }^{\circ} \mathrm{C}$; en las máximas (gráfico $5 \mathrm{~B}$ ) predominan las débiles o muy débiles, con más del $70 \%$ de los casos en que las diferencias no superan los $2{ }^{\circ} \mathrm{C}$.

GrÁFICO 5A. INTENSIDAD DE LA ISLA DE CALOR ESTIVAL EN MADRID, DURANTE LA NOCHE (TEMPERATURAS MÍNIMAS), EXPRESADA EN \% RESPECTO AL TOTAL DE DÍAS CON ISLA DE CALOR (2002-2004)

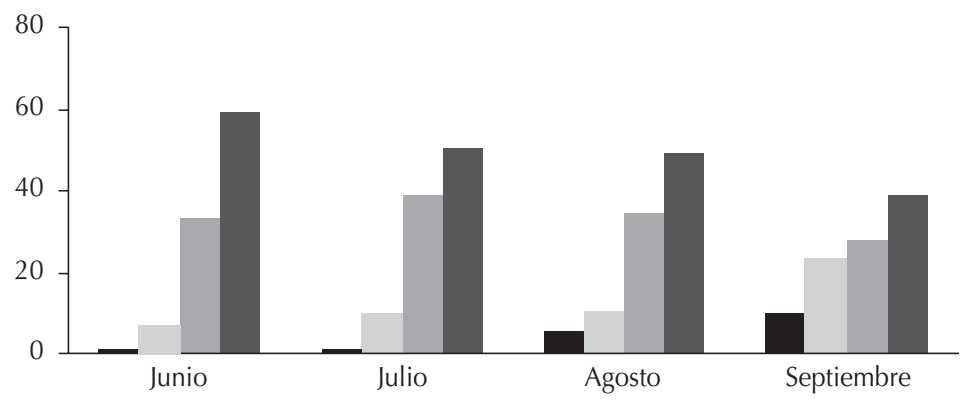

Débil (inferior a $2{ }^{\circ} \mathrm{C}$ )

Moderada $\left(2{ }^{\circ} \mathrm{C}\right.$ a $\left.4{ }^{\circ} \mathrm{C}\right)$

- Fuerte $\left(4^{\circ} \mathrm{C}\right.$ a $\left.6{ }^{\circ} \mathrm{C}\right)$

Muy fuerte (superior a $6{ }^{\circ} \mathrm{C}$ )
1,1

6,7

33,3

58,9
1,1

9,7

38,7

50,5
5,4

10,8

34,4

49,5
10,0

23,3

27,8

38,9

Fuente: Elaboración propia a partir de datos de la red municipal y los del observatorio de Barajas.

GRÁFICO 5B. INTENSIDAD DE LA ISLA DE CALOR ESTIVAL EN MADRID, DURANTE EL DÍA (TEMPERATURAS MÁXIMAS), EXPRESADA EN \% RESPECTO AL TOTAL DE DÍAS CON ISLA DE CALOR (2002-2004)

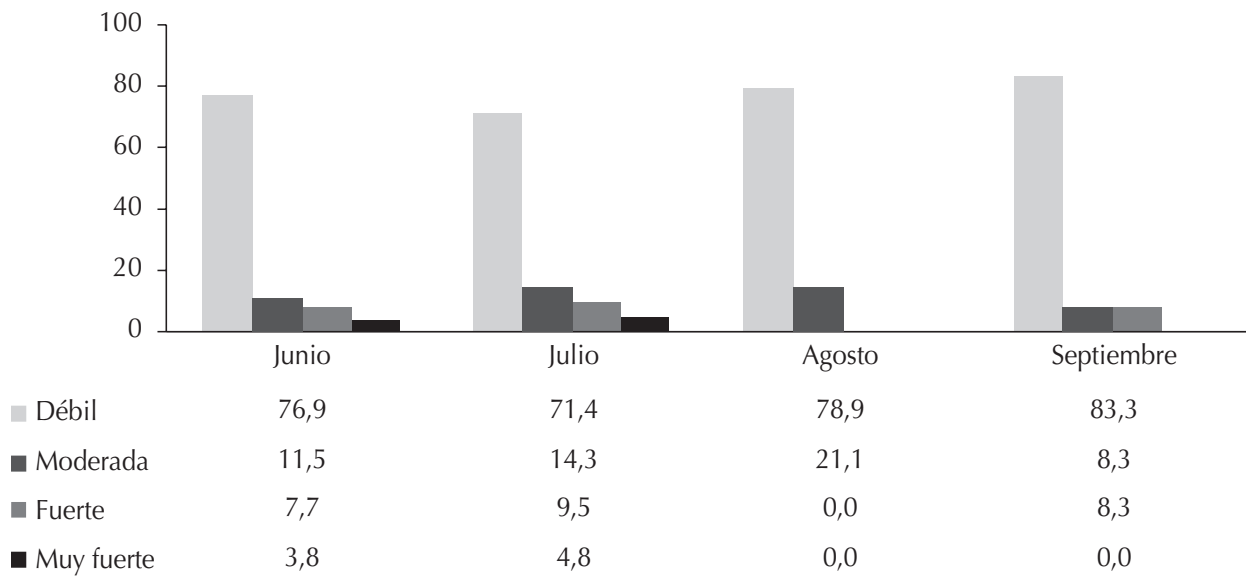

Fuente: Elaboración propia a partir de datos de la red municipal y los del observatorio de Barajas. 
Influencia de la ciudad durante los días cálidos y olas de calor

En el gráfico 6 aparecen representadas las temperaturas máximas y mínimas registradas en un observatorio urbano, la estación 105 (Plaza de Barceló) y las de Barajas, durante todos los días en los que las máximas de este último fueron superiores a $36,5^{\circ} \mathrm{C}$ : se observa que las temperaturas máximas fueron muy similares en ambos observatorios y hay días en los que las temperaturas son más elevadas en el aeropuerto de Barajas; diferente es el comportamiento de las temperaturas mínimas que en la ciudad se mantienen sistemáticamente por encima de los $23^{\circ} \mathrm{C}$, mientras que fuera de ella la mayoría de los días desciende por debajo de los $20^{\circ} \mathrm{C}$.

GrÁFICO 6. TeMPERATURAS MÁXIMAS Y MÍNIMAS EN BARAJAS Y EN LA RED MUNICIPAL, DURANTE LOS DíAS EN QUE LA TEMPERATURA MÁxiMA DE BARAJAS SUPERÓ lOS 36,5 ${ }^{\circ} \mathrm{C}$ (2002-2004)

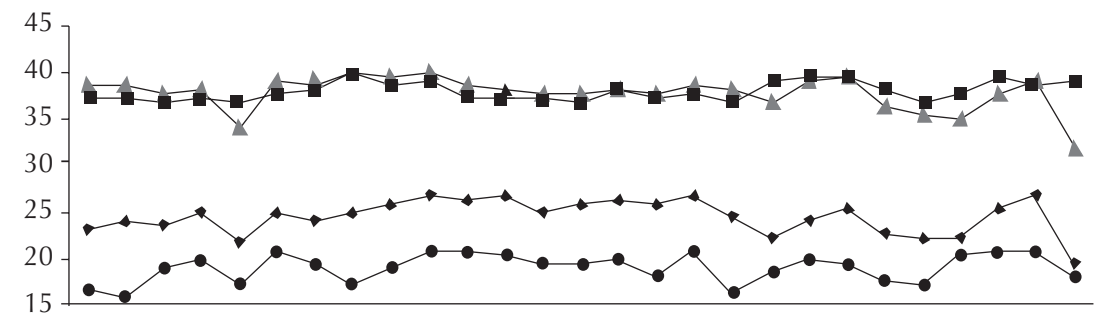

$\begin{array}{llllllllllllllllllllllllllllllll}6,2 & 6,2 & 7,2 & 7,2 & 6,3 & 7,3 & 7,3 & 8,3 & 8,3 & 8,3 & 8,3 & 8,3 & 8,3 & 8,3 & 8,3 & 8,3 & 8,3 & 6,4 & 6,4 & 6,4 & 6,4 & 6,4 & 7,4 & 7,4 & 7,4 & 7,4 & 8,4\end{array}$

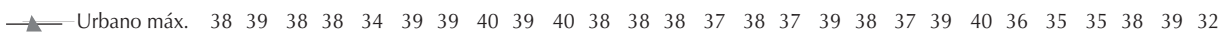

—— Barajas máx. $\begin{array}{lllllllllllllllllllllllllll}37 & 37 & 37 & 37 & 37 & 37 & 38 & 40 & 38 & 39 & 37 & 37 & 37 & 37 & 38 & 37 & 38 & 37 & 39 & 39 & 39 & 38 & 37 & 38 & 40 & 39 & 39\end{array}$

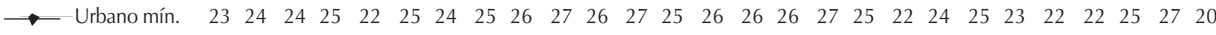

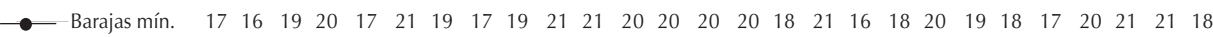

Fuente: Elaboración propia, a partir de datos de la red municipal y los del observatorio de Barajas. En el eje horizontal, el primer número indica el mes y el segundo el año en los que se han superado los $36,5^{\circ} \mathrm{C}$.

Además, si comparamos el total de días con temperaturas máximas superiores a $36,5^{\circ} \mathrm{C}$ vemos que las diferencias son muy pequeñas (gráfico 7), pero esta situación cambia drásticamente cuando comparamos el total de noches cálidas, es decir, aquellas con temperaturas medias superiores a $20{ }^{\circ} \mathrm{C}$ : en la ciudad representan el $60 \%$ de todos los días estivales, frente al $7,8 \%$ de Barajas.

Durante una ola de calor como la de agosto de 2003, el comportamiento térmico de la ciudad y del campo difieren sustancialmente en el periodo diurno y el nocturno, tal y como se muestra en el gráfico 8 . En él se 
GRÁFICO 7. TOTAL DE DÍAS EXTREMADAMENTE CÁLIDOS Y NOCHES CÁLIDAS EN EL OBSERVATORIO urbano de la Plaza de Barceló (estación 105) y en Barajas (2002-2004)

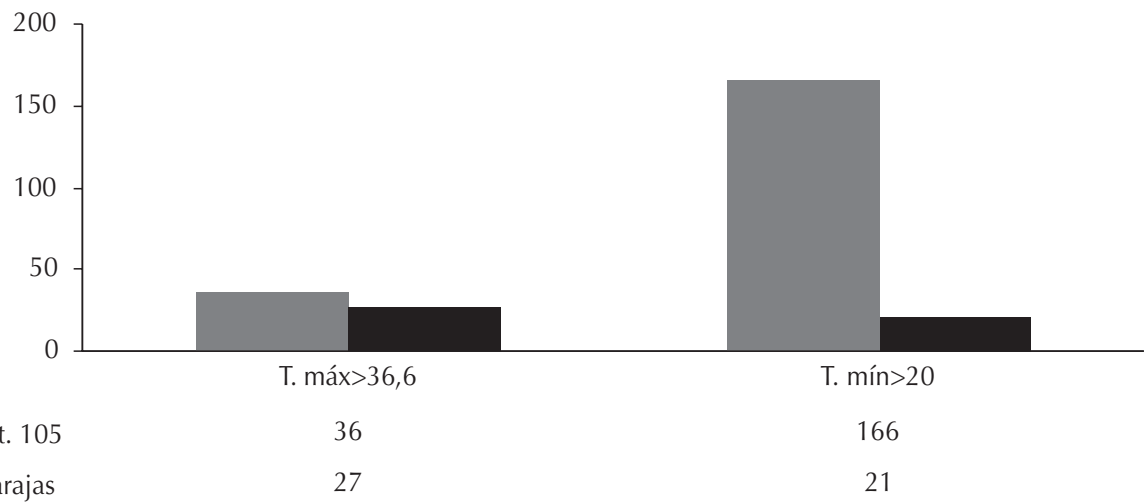

representan los datos horarios de dos estaciones de la red municipal, la 106 y la 105. La primera está situada al oeste en la zona denominada Puente de los Franceses, es una zona de escasas edificaciones, próxima a las grandes masas forestales de la Casa de Campo y el Monte del Pardo; la segunda se localiza en la Plaza de Barceló, en pleno centro urbano y con muy pocos espacios verdes. Se observa como desde las 12 a las 21 horas las temperaturas del Puente de los Franceses se mantienen en torno a $1{ }^{\circ} \mathrm{C}$ más altas que en Barceló y es a partir de las 22 horas cuando el calentamiento urbano se manifiesta de forma clara y continuada: el descenso térmico en la zona no urbanizada es muy acusado y desciende hasta los $17,6^{\circ} \mathrm{C}$; en Barceló el enfriamiento es sincrónico con el anterior, pero mucho más lento y las mínimas sólo descienden hasta las $23,3{ }^{\circ} \mathrm{C}$.

De lo que acabamos de exponer se deducen dos aspectos sumamente interesantes y claves a la hora de evaluar la influencia de la ciudad: el primero, que el efecto urbano durante las olas de calor se manifiesta en un aumento en su duración, más que en una intensificación del calor, puesto que esto último supondría que las temperaturas máximas son mucho más elevadas en la ciudad que en las zonas no urbanas, lo que ocurre sólo en contadas ocasiones; el segundo, que el rasgo fundamental y característico del clima urbano durante estos episodios cálidos es el mantenimiento de un estrés térmico por encima de los niveles de confortabilidad durante las 24 horas del día, lo que agudiza la sensación de disconfort y, por tanto, los efectos negativos sobre la población (Fernández García, 2001-2002). 
GRÁFICO 8. TEMPERATURAS MEDIAS HORARIAS EN DOS ESTACIONES DE LA RED MUNICIPAL DE MADRID, DURANTE LA OLA DE CALOR DE AGOSTO DE 2003

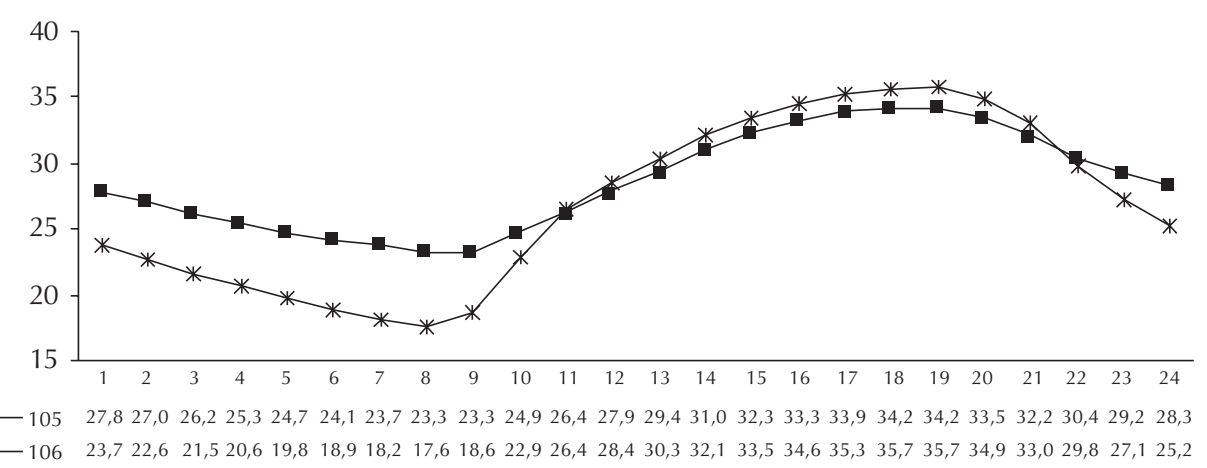

Fuente: Elaboración propia a partir de datos de la red municipal: 105, Plaza de Barceló y 106, Puente de los Franceses.

Todo ello nos conduce a considerar que la temperatura máxima no es un indicador válido para medir la magnitud del mencionado efecto urbano, por lo que es necesario utilizar algún otro que permita incluir las mínimas. El índice conocido como grados día de refrigeración (GDR) podría ser un buen indicador de la intensidad del calor adicional generado por la ciudad y, al mismo tiempo, de las diferencias que aparecen dentro de ella, como consecuencia de la variedad de usos del suelo y de morfologías urbanas.

\section{LOS GRADOS DÍA DE REFRIGERACIÓN (GDR) COMO INDICADOR DEL EFECTO URBANO}

Los grados día de calefacción o refrigeración (GDC, GDR) son los indicadores clásicos que relacionan el gasto energético con el clima y con ellos se determinan los periodos durante los cuales se requiere calefacción o refrigeración en un determinado lugar y el total de energía necesaria para mantener las condiciones de confort aceptables en el interior de los edificios. En el, ya mencionado, Código Técnico de la Edificación se utilizan como base de la normativa que han de cumplir los edificios para el ahorro energético y el Instituto de Recursos Mundiales (Baumet y Selman, 2003; World Resources Institute, 2008), los incluye entre los indicadores climáticos del calentamiento global (Climate analysis Indicators Tools, CAIT). 
El periodo de calefacción se define como aquel durante el cual las temperaturas se mantienen por debajo del umbral de confort, en cuyo caso hay que aumentar la temperatura interior; el de refrigeración como el periodo durante el cual las temperaturas superan el umbral de confort, en cuyo caso es preciso disminuir la temperatura. La suma acumulada durante cualquiera de estos periodos de los grados de temperatura, por encima o por debajo de los umbrales, es un buen indicativo del gasto total de energía potencialmente consumida en un determinado lugar y, lógicamente, de las diferencias térmicas entre diferentes lugares (Fernández García, 1996).

En este trabajo, como temperatura umbral hemos seleccionado $20^{\circ} \mathrm{C}$, siguiendo la metodología propuesta por el CTE y los grados día de refrigeración (GDR) los hemos obtenido a partir de la temperatura media diaria, siempre y cuando el resultado fuese un valor positivo.

\section{Grados día de refrigeración (GDR) y efecto urbano}

Con el fin de diferenciar el efecto urbano en periodos climáticamente normales del que se produciría con olas de calor o días de calor extremo, hemos calculado tres índices: el primero, al que hemos denominado grados día de refrigeración medio (GDR-md), incluye todos los días de junio, julio, agosto y septiembre de 2002 a 2004; el segundo, al que hemos denominado grados día de refrigeración en días cálidos (GD-dcal), sólo se han considerado aquellos días en los que la temperatura máxima de Barajas fue superior a $36,5^{\circ} \mathrm{C}$ y el tercero, GDR-ola, se ha obtenido teniendo en cuenta sólo los periodos considerados como olas de calor.

En el cuadro 6 se recogen los valores medios diarios de los GDR en cada uno de los observatorios de la red municipal y en Barajas. La media diaria de GDR-md oscilan entre 90 y 162; en situaciones extremadamente cálidas y durante las olas de calor, la media diaria lógicamente aumenta, oscilando entre los 161 y los 260, en el primer caso y entre 210 y 299 con olas de calor (cuadro 7). Los máximos siempre corresponden al observatorio con rasgos urbanos más claros, el 105 correspondiente a Barceló y los mínimos a Barajas y algunas estaciones de la red urbana, pero en situación periférica y en zonas de urbanización muy escasa. Los valores más bajos se dan en la estación 106, Puente de los Franceses, como consecuencia, no sólo de la débil urbanización del entorno donde se ubica, sino que además influyen otros factores como es la proximidad de las dos grandes superficies boscosas que rodean Madrid (la Casa de 
Campo y el Monte del Pardo) y su emplazamiento en la vaguada del Manzanares por donde penetran las masas de aire frío procedentes del norte y que convierten a esta zona de la ciudad en la más fría durante todas las épocas del año (Fernández García, López Gómez y Almendros, 1994).

Cuadro 6. Media diaria de los GDR en las estaciones de la ReD mUNICIPAL y EN BARAJAS DURANTE EL VERANO (2002-2004)

\begin{tabular}{lccccccc} 
GDR & Est. 100 & Est. 101 & Est. 102 & Est. 105 & Est. 106 & Est. 107 & Barajas \\
\hline Media & 102 & 118 & 98 & 162 & 90 & 114 & 110 \\
Máx. 36,5 & 197 & 206 & 192 & 260 & 161 & 195 & 200 \\
Ola calor & 210 & 240 & 200 & 299 & 210 & 235 & 219
\end{tabular}

Fuente: Elaboración propia a partir de datos de la red municipal y los del observatorio de Barajas.

El calentamiento provocado por la ciudad es claro, pero además se observa como este efecto se agudiza a medida que lo hace el calor. Así las diferencias de GDR entre la estación urbana más cálida y Barajas pasan de 52 en situaciones normales, a 60 en los días extremadamente cálidos y alcanzan los 80 GDR durante las olas de calor gráfico 9).

Gráfico 9: Diferencias en GDR eNtre Barajas y Barceló (eStaCión 105), en VeRANO (GDR-MD), EN LOS DÍAS EXTREMADAMENTE CÁLIDOS (GDR-36,5) Y DURANTE LOS EPISODIOS DE OLA DE CALOR (GDR-OLA)

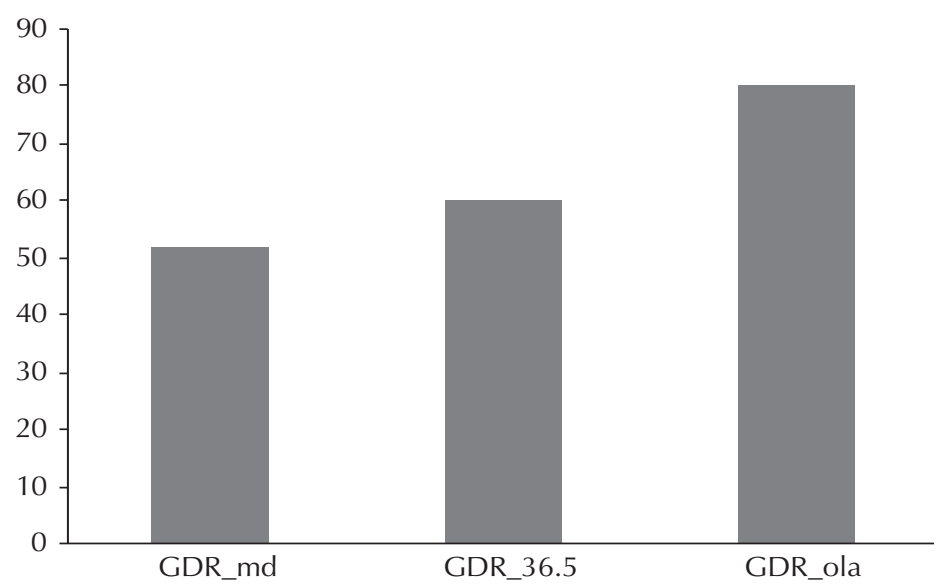

Urb-rural

52

60

80 


\section{Diferencias intraurbanas: influencia de los usos del suelo}

La ciudad no es un espacio homogéneo y lo que denominamos genéricamente como clima urbano presenta gran cantidad de matices asociados a la morfología de los edificios, el trazado de las calles y la presencia de zonas verdes. Los estudios realizados en Madrid a partir de los datos recogidos en más de 160 transectos térmicos (Fernández García, F., Montávez, P. et al., 2003), ponen de manifiesto que los dos factores que de manera más directa condicionan la isla de calor en nuestra ciudad son la densidad de edificaciones y la presencia de espacios verdes. Como avance de los estudios actualmente en curso sobre la relación de las temperaturas y usos del suelo presentamos los resultados de combinar la media diaria de GDR durante la ola de calor de agosto de 2003 y los principales usos del suelo de Madrid y su área metropolitana. Para ello, hemos introducido los observatorios de las tres redes de observación existentes en nuestra zona (la municipal, la de contaminación y la oficial de la AEMET, citadas anteriormente) y con ellas hemos obtenido, por interpolación, el mapa 2 de GDR en Madrid y su área metropolitana. Hemos utilizado el método Kriging de interpolación del módulo Spacial Analyst del SIG ArcView 9.2 y con el álgebra de mapas hemos combinado los resultados con los usos del suelo.

La distribución espacial de los GDR durante la ola de calor de agosto de 2003 muestran dos zonas con máximos superiores a los 300 GDR, que se corresponden con áreas de fuerte urbanización (mapa 2): una, en el centro de la ciudad, en los aledaños de la estación de Barceló y, la otra, en los barrios del suroeste, en torno a las estaciones de General Ricardos y Plaza Elíptica. Los mínimos, inferiores a 200 GDR, se localizan al noroeste, coincidiendo con los observatorios de Majadahonda, Puente de los Franceses y Fuencarral, todos ellos en zonas de escasa urbanización y próximos a las grandes masas boscosas del Monte del Pardo y la Casa de Campo. Hacia el suroeste el descenso es menos acusado, por su menor altitud, la ausencia casi total de grandes masas arbóreas y, sobre todo, por la presencia de grandes núcleos suburbanos, como Alcorcón y Villaverde.

La influencia de la urbanización es clara, tal y como se puede apreciar en el gráfico 10, obtenido al comparar los valores interpolados y las principales estructuras urbanas de Madrid. La ocupación del espacio rural y su transformación en tejido urbano, es la principal causa del recalentamiento; a su vez, la mayor o menor densidad de edificaciones y la presencia de zonas arboladas introducen importantes matices, cuya consideración a la hora de planificar la ciudad, podría mejorar considerablemente las condiciones ambientales de los espacios urbanos. 
MAPA 2. DistribuCión ESPACIAL DE LA MEDIA DIARIA DE LOS GRADOS DÍA DE REFRIGERACIÓN (GDR), DURANTE LA OLA DE CALOR DE AGOSTO DE 2003

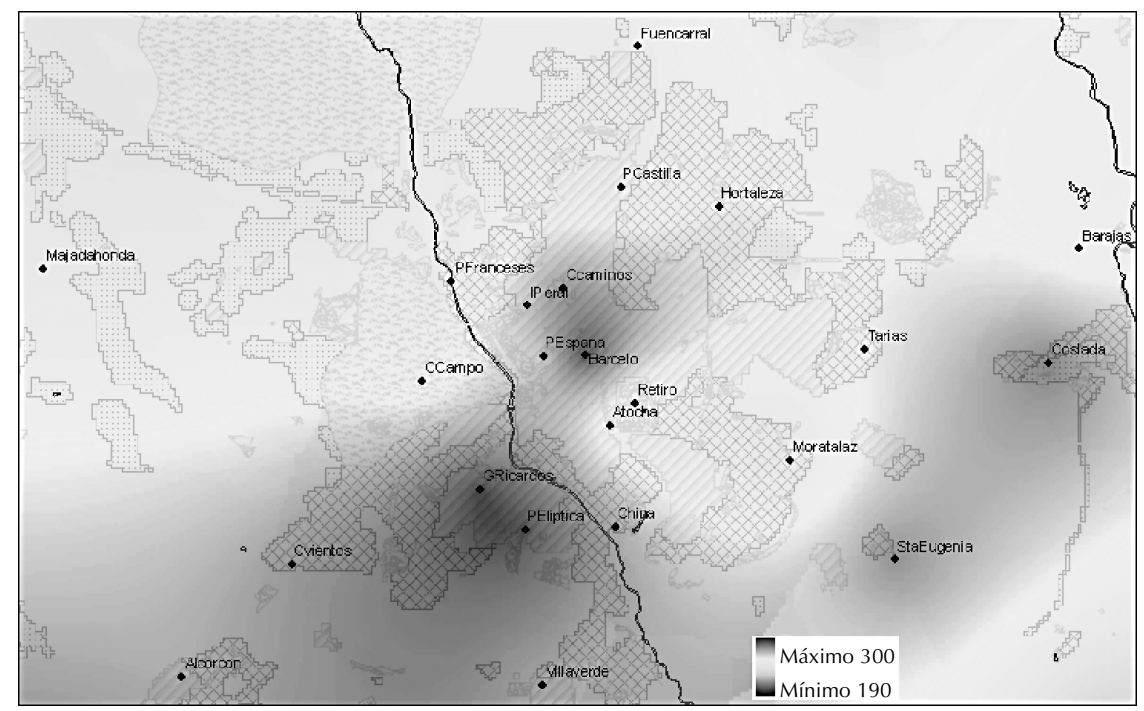

Gráfico 10. Promedio diario de los GDR, durante la ola de calor de aGosto DE 2003, EN DIFERENTES ESTRUCTURAS URBANAS

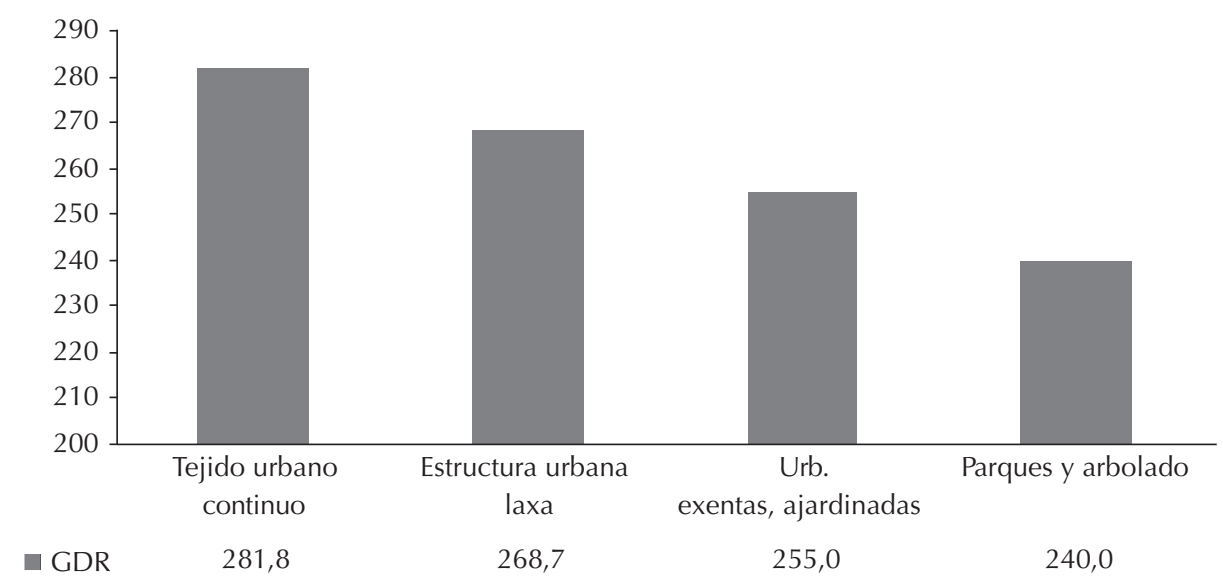

\section{Conclusiones:}

- Las olas de calor constituyen un fenómeno frecuente en Madrid y su duración y frecuencia ha experimentado una tendencia creciente a partir de 1981, aunque su intensidad se ha mantenido estable. 
- La influencia urbana se manifiesta principalmente por el aumento de la duración de estos periodos excepcionalmente cálidos, pero no de su intensidad, ya que durante el día las temperaturas máximas se mantienen muy similares tanto en la ciudad como en las zonas extraurbanas. Es durante la noche cuando las diferencias se acentúan, manteniéndose las temperaturas mucho más altas en el interior de la ciudad, hasta triplicar el número de noches cálidas, respecto a las áreas periféricas.

- Como consecuencia de lo expresado en el párrafo anterior, para evaluar el efecto urbano es preciso recurrir a índices, como los grados día de refrigeración (GDR), que integran, tanto las temperaturas máximas como las mínimas. El empleo de este índice nos muestra que en el caso de Madrid, el efecto urbano es tanto mayor cuanto más elevadas son las temperaturas, de tal modo que en situaciones normales es de 52 GDR, pero alcanza los 80 durante las olas de calor.

- La morfología urbana y, en general, los diferentes usos del suelo, constituyen un factor determinante a la hora de explicar las diferencias espaciales en la magnitud del calor dentro de la misma ciudad. La prolongación del calor nocturno es máxima en ámbitos urbanos de alta densidad edificatoria y escasos espacios verdes, habitados normalmente por población envejecida, de nivel de renta bajo y muy vulnerable ante estos episodios térmicos extremos. Es, por tanto, un factor esencial a tener en cuenta, tanto en el diseño de los planes de prevención ante fenómenos de esta naturaleza, como en las actuaciones de remodelación de los barrios degradados.

La ciudad es un espacio complejo, donde las condiciones térmicas pueden variar muy rápidamente y de forma notoria en zonas muy próximas en función de los materiales que conforman el tejido urbano, las sombras generadas por los edificios, o la velocidad del viento, muy variables en función del trazado y orientación de las calles. Además, las sensaciones térmicas dependen, no sólo de la temperatura del aire, sino también de la radiación emitida por el asfalto y las paredes de los edificios (la denominada temperatura radiante), de la humedad y el viento. Conocer como interactúan todos estos mecanismos a escala de cañón urbano, el espacio realmente vivido por los habitantes de la ciudad, es el reto que tiene planteada la climatología urbana actual. Este trabajo es, por tanto, sólo una aproximación a un problema mucho más complejo en el que es preciso seguir profundizando.

Recibido 2.09.08

Aceptado 10.11.08 


\section{BIBLIOGRAFÍA}

Alberdi, J. C.; Díaz J.; Montero, J. C. y Miron, I. (1998): Daily mortality in Madrid community 1986-1992: relationship with meteorological variables, Eur J Epidemiol, 14, pp. 571 578

Baumet, K. y Selman, M. (2003): "Heating and cooling degree days", Worl Resouces Institute; disponible en: http://cait.wri.org

CAM (2006): Vigilancia y control de los efectos de las olas de calor. Plan de respuesta ante los riesgos, Instituto de Salud Pública, Servicio de Promoción de la Salud, Madrid, documentos técnicos, $30 \mathrm{pp}$.

Coppe, C. et al. (2004): "Health and Global Environmental Change.Heat-waves: risks and responses", World Health Organization, $80 \mathrm{pp}$.

Díaz J.; García-Herrera, R.; Trigo R. M.; Linares, C.; Valente, M. A. y Hernández, E. (2006): "The impact of summer 2003 heat wave in Iberia: how should we measure it?", International Journal of Biometeorology, 50, pp. 159-166.

Díaz, J.; Linares, C. y Tobías A. (2006): "Impact of extreme temperatures on daily mortality in Madrid (Spain) among the 45-64 age-group", International Journal of Biometeorology, 50 (6), pp. 342-348.

Díaz, J.; Jordan, A.; García, R.; López, C.; Alberdi, J. C.; Hernández, E. y Otero, A. (2002): "Heat waves in Madrid 1986-97: effects on the health of the elderly", International Archives of Occupational and Environmental Health, 75, pp. 163-70.

Desirex 2008 Campaign: Experiment Plan. Prepared by: José A. Sobrino, University of Valencia (Spain). Date: 7 June 2008. Status: Working document.

Ebi, K. L.; Teisberg, T. J.; Kalkstein, L. S.; Robinson, L. y Weiher, R. F. (2004): "Heat Watch/Warning Systems Save Lives: Estimated Costs and Benefits for Philadelphia 19951998", Bulletin of the American Meteorological Society, 85, pp. 1067-74.

Fernández García, F. (1995): Manual de Climatología Aplicada. Clima, Medio Ambiente y planificación, Síntesis, Madrid, 216 pp.

Fernández García, F.; Almendros Coca, M. A., y López Gómez, A. (1994): "La influencia del relieve en la Isla de calor de Madrid: las vaguadas del manzanares y el Albroñigal", Revista de Estudios Geográficos, no 224, pp. 473-494.

Fernández García, F. (2001-2002): "El clima urbano de Madrid y su influencia sobre el confort térmico", Boletín de la Real Sociedad Geográfica, CXXXVII-CXXXVIII, pp. 169-185.

Fernández García, F.; Montávez, J. P.; González-Rouco, F. J. y Valero, F. (2003): "A PCA analisys of the UHI form of Madrid (Spain)", 5th International Conference on Urban Climate, 1-5/9, Lodz, Poland.

Fernández García, F. y Moreno Jiménez, A. (2004): “Confort climático y nivel de renta en la Comunidad de Madrid: un estudio exploratorio de su relación espacial", Historia, Clima y Paisaje. Estudios geográficos en memoria del profesor Antonio López Gómez, Ed. Universitat de Valencia, Universidad Autónoma de Madrid y Universitat de Alicante, Valencia, pp. 305-321.

Fernández García, F. (2005): “Contaminación atmosférica y calidad del aire en Madrid: análisis de las concentraciones de $\mathrm{SO}_{2}, \mathrm{CO}$, Ozono y PM10 (1980-2003)", Revista Estudios Geográficos, LXVI, 259, pp. 507-532.

Fernández García, F. (2007): "Impactos del cambio climático en las áreas urbanas y rurales", Boletín de la Institución Libre de Enseñanza, nº 66-67.

García Herrera, R.; Prieto, L.; Díaz J.; Hernández, E. y Del Teso, T. (2002): "Synoptic conditions leading to extremely high temperatures in Madrid", Annales Geophysicae, 20 (2), pp. 237-245.

García-Herrera, R.; Díaz, J.; Trigo, R. M. y Hernández, E. (2005): "Extreme summer temperatures in Iberia: health impacts and associated synoptic conditions", Annales Geophysicae, 23, pp. 239-251. 
Kalkstein, L. et al. (1996): "The Philadelphia hot weather-health watch/warning system: development and Application Summer 1995", Bulletin of the American Meteorological Society, 77, pp. 1519-28.

Kalkstein, L. S.; Greene, J. S.; Mills, D. y Perrin, A. (2008): "The Development of Analog European Heat Waves for U.S. Cities to Analyze Impacts on Heat-Related Mortality", Bulletin of the American Meteorological Society, submitted.

Kjellström, E.; Bärring, L.; Jacob, D.; Jones, R.; Lenderink, G. y Schär, C. (2007): "Modelling daily temperature extremes: recent climate and future changes over Europe", Climatic Change, 81, pp. 249-265.

López Gómez, A. y Fernández García, F. (1984): "La isla de calor en Madrid: avance de un estudio de clima urbano", Revista de Estudios Geográficos, n 174, pp. 5-34.

López Gómez, A.; Fernández García, F. y Moreno Jiménez, A. (1993): El clima urbano. Teledetección de la isla de calor en Madrid, Ministerio de Obras públicas y Transportes, Madrid, Serie Monografías, 230 pp.

MIMAM (2005): Evaluación preliminar de los Impactos en España por Efecto del Cambio Climático, en Moreno Rodríguez, J. M. (coord.), 822 pp.

MMA (2007): "El cambio climático en España. Estado de situación", Informe para el presidente del gobierno elaborado por expertos en cambio climático, Documento resumen, noviembre de 2007, en: http://www.mma.es/portal/secciones/cambio_climatico/

MMA (2008): Primer informe de seguimiento sobre el desarrollo del Plan Nacional de Adaptación al Cambio Climático, Oficina Española del Cambio Climático, 63 pp.

Rasilla Álvarez, D. y Fernández García, F. (2005): “Trends on extreme temperature days over the Iberian peninsula", $17^{\text {th }}$ International Congress of Biometeorology. ICB 2005. September 05-09, 2005. Gamisch-Partenkirchen (Germany).

Sánchez, E.; Gallardo, C.; Gaertner, M. A.; Arribas, A. y Castro, M. (2004). "Future climate extreme events in the Mediterranean simulated by a regional climate model: a first approach", Global and Planetary Change, 44(1-4), pp. 163-180.

Sheridan, S. C. y Kalkstein, L. S. (2004): "Progress in Heat Watch-Warning System Technology", Bulletin of the American Meteorological Society, 85, pp. 1931-41.

Taha, H.; Kalstein, L.; Sheridan, S. y Wong, E. (2005): "Impacts of large-scale surface modifications on meteorological conditions and energy use: A 10-region modeling study", Theoretical and Applied Climatology, vol. 62, pp. 175-185 (1999).

Valor, E.; Meneu, V. y Caselles, V. (2001): "Daily Air Temperature and Electricity Load in Spain", Journal of Applied Meteorology, 40(8), pp. 1413-1421.

Worl Resouces Institute (2008): CAIT: indicator framework paper, disponible en: http://cait.wri.org

\section{Resumen}

La frecuencia y duración de los episodios cálidos extremos, un fenómeno habitual en el clima mediterráneo continental de la Meseta Meridional, ha aumentado en el área de Madrid desde los años noventa, aunque su magnitud permanece constante. En el interior de la aglomeración urbana, el efecto general de la ciudad sobre estos eventos climáticos extremos ha supuesto una exacerbación del calor, no tanto por un aumento de la temperatura máxima, como por una persistencia del calor en las horas nocturnas. No obstante, la diversidad de la morfología urbana introduce variaciones espaciales en la intensidad de este calor nocturno, agravando sus efectos en las áreas densamente urbanizadas y mitigándolos en las proximidades de las áreas verdes.

Palabras clave: Eventos cálidos extremos; isla de calor urbana; usos del suelo; grados día de refrigeración (GDR); Madrid (España). 


\section{Abstract}

The frequency and duration of the warmest temperature episodes, a common phenomenon under the continental Mediterranean conditions of the Spanish Southern Meseta, has increased in the Madrid area since the 80's, although their magnitude remains unchanged. The effect of the urban environment on those extreme events has exacerbated the heat load due to the persistence of the high temperatures along the night time hours. Nevertheless, the diversity of the urban morphology introduces a spatial variability on the strength of this nocturnal heat load, aggravating it in the densely urbanized areas and mitigating it in the vicinities of the green areas.

Key words: Extreme warm events; urban heat island; land use; cooling degree day (CDD); Madrid (Spain).

Agradecemos a Francisco Moya, jefe de sección de Información Ambiental del Ayuntamiento de Madrid, las facilidades en la obtención de los datos y su valiosa información sobre la red meteorológica municipal. 